\title{
Artificial intelligence of ophthalmology ushers in a new stage of the challenging times
}

Prof. Lezheng $\mathrm{Wu}, \mathrm{MD}$

\section{Correspondence to: Prof. Lezheng Wu, MD}

President of Afro-Asian Council of Ophthalmology, Zhongshan Ophthalmic Center (ZOC), Sun Yat-Sen University, Guangzhou 510060, China. lechwu@hotmail.com

Received: 3-1-2021, Accepted: 3-3-2021, Published online:11/3/2021

EJO(MOC) 2021;1:55-57.

Short title: Artificial intelligence of ophthalmology.

\section{Introduction}

As a symbol of the rapid development of science and technology in world nowadays, artificial intelligence (AI) technology and its endless applications are profoundly affecting the pattern of human survival and development in all aspects. AI technology is an intelligent tool created by human beings, which has greatly changed people lives and become one of the most active new elements. AI permeates all aspects of human life, including medical science. The application of AI technology in ophthalmology is already on the agenda (Figure 1), and it is making continuous progress in practice and application, making leaps and bounds.

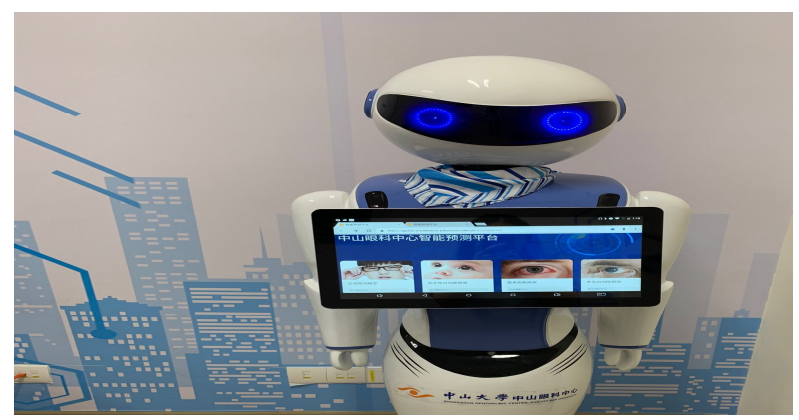

Figure 1: An intelligent diagnosis and prediction robot for eye diseases developed by the AI team of Zhongshan Ophthalmic Center (ZOC), Sun Yat-sen University, China

\section{Ophthalmic AI is also characterized by big} data

After big data processing, visual function measurement can achieve maximum accuracy and standardization. The whole visual system, from the eyeball to the brain, not only has information about organs and tissues, such as the position, size, shape and rotation of the eyeball, but also has quite prominent histological features, such as iris, fundus blood vessels, etc. With the process of big data, the boundaries between normal and abnormal as well as the pathological or normal boundaries can be normalized and identified respectively. Especially in the transmission of visual information, digitization becomes the most basic and successful information source. In terms of visual information, such as electroretinogram (ERG), VEP (Visual Evoked Potential) of optic nerve and visual cortex

Egyptian Journal of Ophthalmology, a publication of Mansoura Ophthalmic Center.

Address: Mansoura Ophthalmic Center, Mansoura University, Mansoura, Egypt.

Website: https://ejomos.journals.ekb.eg

Tel. 0020502202064 Fax. 0020502202060.

E-mail: ejo@mans.edu.eg 
information, as well as EOG (Electro-Oculogram) and EMG (Electromyography) of eye movements, all vital sources of visual information. In addition, the length of the eye axis and the shape of the eyeball are also important sources of information. In ophthalmic epidemiology, big data is more essential for the occurrence, development and prognosis assessment of major eye diseases.

\section{AI is used for image analysis to provide more} accurate judgment for the diagnosis of eye diseases

A large number of eye diseases depend on image analysis provided by a variety of diagnostic tools. Take ocular fundus diseases as an example, imaging plays a more important role, and artificial intelligence can play an important role in screening and accurate diagnosis through image analysis. At present, vascular diseases represented by hypertension and metabolic diseases represented by diabetes have important characteristics of fundus changes. The main structures that constitute the fundus manifestations: optic disc, blood vessels, various parts of the retina, as well as many of the observed manifestations under the cooperation of fluorescent angiography and other examinations, all have image and digital characteristics, which can become the control and monitoring conditions of related diseases, and provide the basis for early intervention to develop early control and early treatment strategies. The application of AI enables the vast majority of patients with fundus diseases to get intelligent diagnosis and formulate more effective treatment strategies and measures, and also benefits the training of younger ophthalmologists and alleviates areas in which facing shortage of medical services and medicines, proving advantages of AI technology.

\section{The era of robotic ophthalmic surgery}

The emergence and application of robotic ophthalmic surgery is a significant progress in the technological innovation and development of artificial intelligence medical technology in ophthalmology. By the 21st century, this field has become an important field of practice in ophthalmic surgery. Robotic surgery has many advantages, such as accuracy and control. Compared with traditional surgeons, robots are more accurate in spatial resolution, depth perception, tactile feedback, and so on, and are more conducive to rule out physiological hand shaking and other factors, which are more challenging in the surgical requirements of fine ophthalmic operation. Robotic ophthalmic surgery is also constructive to teaching, training young doctors, can be used for remote guidance, and has been applied in corneal surgery, cataract extraction , retinal vein cannulation and so on.

IV. AI technology promotes the accuracy of

\section{biometrics}

As an organ with important information content, 
the eye plays an important role in biometrics, like many biological characteristics of the human body: face, fingerprints, voice lines, body temperature, and so on. , coupled with walking gait, sitting posture, signature handwriting and other behavioral characteristics, there are many personalized information content of the human body, of which there are also specialized tissues involving the eyeball, such as the iris, fundus vessels — arteries and veins. This information is greatly conducive to application in medical diagnosis, as well as industry and management. Bio-metrics is closely related to digitization in the era of artificial intelligence. In ophthalmic tissues, from iris structure to further analysis of fundus vessels, it is gradually more prominent, which may involve new understanding of fine nerves, micro-vessels, micro-circulation and other fields in the prevention of blindness in some important eye diseases, and will take a new step in the exploration of preventing visual organ damage or reconstruction.

\section{Conclusion}

As one of the core forces driving new science and technology in world nowadays, AI technology and its endless variety of new applications have exerted a profound influence on the development of human society.
The application of AI in ophthalmology is also a challenge of the new era. In order to better protect the visual organs, AI has opened up a new front-line. We will witness new developments in ophthalmology more capable of bringing benefits towards mankind.

\section{References}

1. Long E, Lin H, Liu Z, Wu X, Wang L, Jiang J, et al. An artificial intelligence platform for the multihospital collaborative management of congenital cataracts, Nature - Biomedical Engineering. 2017;1:1-8.

2. Long E, Liu Z, Xiang Y, Xu A, Huang J, Huang X, et al. Discrimination of the behavioural dynamics of visually impaired infants via deep learning, Nature - Biomedical Engineering. 2019;3:860869.

3. Li WT, Yang YH, Zhang K, Long E, He L, Zhang L, et al. Dense anatomical annotation of slit-lamp images improves the performance of deep learning for the diagnosis of ophthalmic disorders, Nature - Biomedical Engineering 2020;4:767-777.

4. Zhu GY, Zhang JS, Wan XH, et al: Ophthalmology Robot Technology. Int Rev Ophthalmol. 2018;42:213-216. 